\title{
19. CONVECTION ZONES AND CORONAE OF WHITE DWARFS
}

\author{
K. H. BÖHM and J. CASSINELLI \\ Astronomy Dept., University of Washington, Seattle, Wash., U.S.A.
}

\begin{abstract}
Outer convection zones of white dwarfs in the range $5800 \mathrm{~K} \leq T_{\text {eff }} \leq 30000 \mathrm{~K}$ have been studied assuming that they have the same chemical composition as determined by Weidemann (1960) for van Maanen 2. Convection is important in all these stars. In white dwarfs $T_{\text {eff }}<8000 \mathrm{~K}$ the adiabatic temperature gradient is strongly influenced by the pressure ionization of $\mathrm{H}, \mathrm{He}$ I and $\mathrm{He}$ II which occurs within the convection zone. Partial degeneracy is also important.

Convective velocities are very small for cool white dwarfs but they reach considerable values for hotter objects. For a white dwarf of $T_{\text {eff }}=30000 \mathrm{~K}$ a velocity of $6.05 \mathrm{~km} / \mathrm{sec}$ and an acoustic flux (generated by the turbulent convection) of $1.5 \times 10^{11} \mathrm{erg} \mathrm{cm}^{-2} \mathrm{sec}^{-1}$ is reached. The formation of white dwarf coronae is briefly discussed.
\end{abstract}

During the last 3 years it has become evident that the existence of outer convection zones of cool white dwarfs strongly reduces the core temperatures, the energy contents and consequently the remaining lifetimes of these objects. Preliminary calculations of convection zones have been carried out by van Horn (1970) and by myself $(1968,1969)$.

It has been pointed out by Greenstein (1969) that the change in the cooling times can become very large because of a feedback between the lowering of the core temperature by convection and the reduction of the specific heat below the Debye temperature.

All earlier calculations of white dwarf convection zones are based on very crude approximations with respect to the thermodynamics. It should be mentioned that in the cool white dwarfs the hydrodynamic aspect does not cause as much trouble as in other stars: The densities are usually so high that one gets an almost sudden transition from a pure radiative to an almost adiabatic gradient.

What kind of improvements are required in the thermodynamics? All previous calculations have used the assumptions that we have a perfect partially ionized gas in the convection zone and that the ionization is governed by the Saha equation.

However, it is clear now that the following complications certainly do arise:

(1) Relatively high (though not complete) electron degeneracy does occur within the convection zones.

(2) $\mathrm{H}$ and $\mathrm{HeI}$ are completely and $\mathrm{He}$ II is partially pressure ionized within the convection zone. The usual Saha equation is not an acceptable approximation.

We have now carried out calculations in which we take into account these two complications:

Pressure ionization is treated according to the Stewart and Pyatt (1966) theory. If applied in a naive way this theory leads to a discontinuity in the entropy and to a singularity of the adiabatic gradient at the point where the ionization becomes complete. In order to avoid this problem we have followed a suggestion by Rouse (1964) which permits a rapid but smooth transition to the state of complete pressure ionization. 
The following effects of partial degeneracy of the electron gas have been taken into account:

(1) The equation of state has been determined assuming that the atoms and ions are nondegenerate but permitting a partial degeneracy of the electrons.

(2) The influence of this degeneracy on the entropy $S$, the adiabatic gradient $\nabla_{\mathrm{ad}}$, the scale height $H$ has been taken into account.

(3) In the mixing length theory one assumes usually that temperature and density fluctuations are related according to

$$
\delta \varrho / \varrho=-\delta T / T
$$

as follows from the assumption of constant pressure within a horizontal plane. We have written

$$
\delta \varrho / \varrho=-D(\delta T / T)
$$

where $D$ is now a complicated factor depending on $T, \varrho$, the degeneracy parameter $\alpha$ and the Fermi Dirac functions.

Numerical calculations have been carried out for white dwarfs of the Weidemann chemical composition (i.e. consisting mostly of $\mathrm{He}$, with $2 \% \mathrm{H}$ and a considerable underabundance of heavier elements) in the range $5800 \mathrm{~K} \leqslant T_{\text {eff }} \leqslant 30000 \mathrm{~K}$. We have used a surface gravity of $10^{8}$ which corresponds to white dwarfs of $0.6 \mathrm{M}$ according to the Chandrasekhar (1939) mass-radius relation.

One of the important results of these calculations is the following: For white dwarfs with $T_{\text {eff }}<8000 \mathrm{~K}$ the adiabatic gradient is strongly influenced by the presence of the three pressure ionization zones for $\mathrm{H}, \mathrm{He}$ I and $\mathrm{He}$ II. In these regions the adiabatic gradient drops to very low values. The net effect of this behavior is of course a lowering of the average adiabatic gradient.

This leads (as compared to the earlier simple models) to a higher degree of degeneracy for a given temperature at the bottom of the convection zone. Consequently we get an even lower core temperature than predicted earlier. A relatively small part of this effect is compensated by the influence of partial degeneracy.

The effect is illustrated in Figure 1 which show the depth dependence of the adiabatic and the radiative gradients in a white dwarf convection zone. We can clearly see the three distinct pressure ionization zones for $\mathrm{H}, \mathrm{HeI}$ and $\mathrm{He}$ II. In a star like van Maanen 2 we get two separate convection zones the lower of which reaches a density of $8.015 \times 10^{2} \mathrm{~g} \mathrm{~cm}^{-3}$ and degeneracy parameter $\alpha=-\mu / k T$ of -13.999 .

It is interesting to note that all white dwarfs up to an effective temperature of $30000 \mathrm{~K}$ have an outer convection zone. This means that convection should be taken into account in practically all studies of white dwarf atmospheres and of the internal thermal structure of these stars.

Figures 2, 3 and 4 show the depth of the convection zone, the temperature and the degeneracy parameter $\alpha$ at the lower boundary of the zone. These figures indicate that convection is much more important for the thermal structure of relatively cool white dwarfs than for hot white dwarfs. This conclusion is based on the following results: 


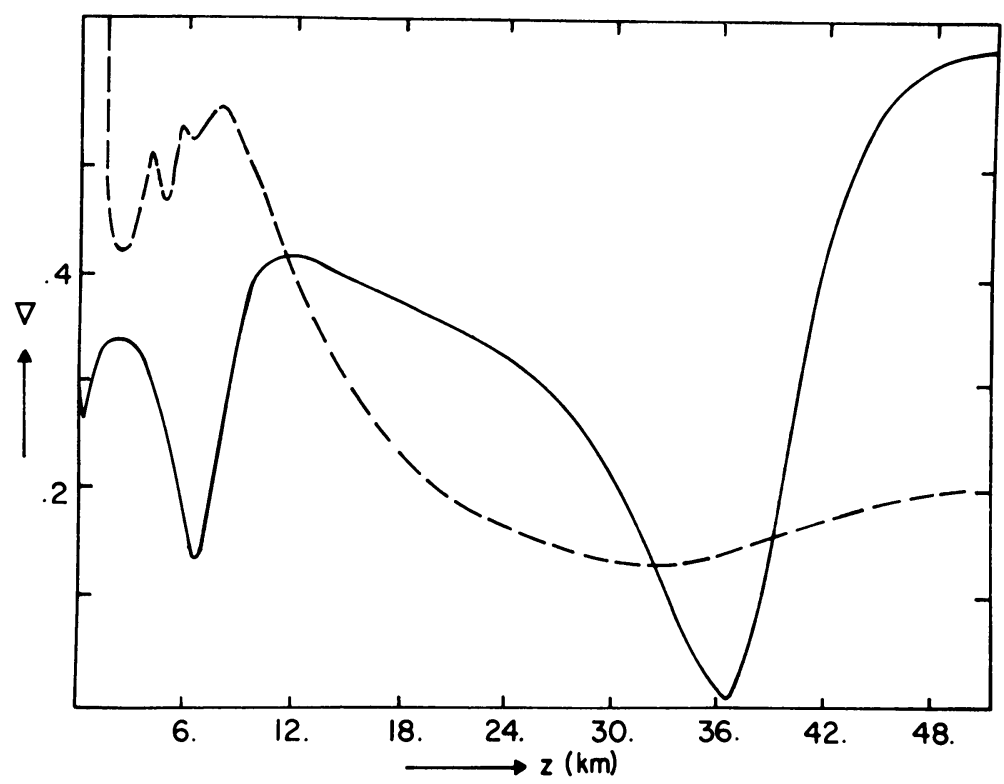

Fig. 1. The adiabatic (solid curve) and the radiative (broken line) gradient as a function of geometrical depth for a white dwarf with $T_{\text {eff }}=6000 \mathrm{~K}, g=10^{8} \mathrm{~cm} \mathrm{sec}^{-2}$. (From Böhm, 1970.)

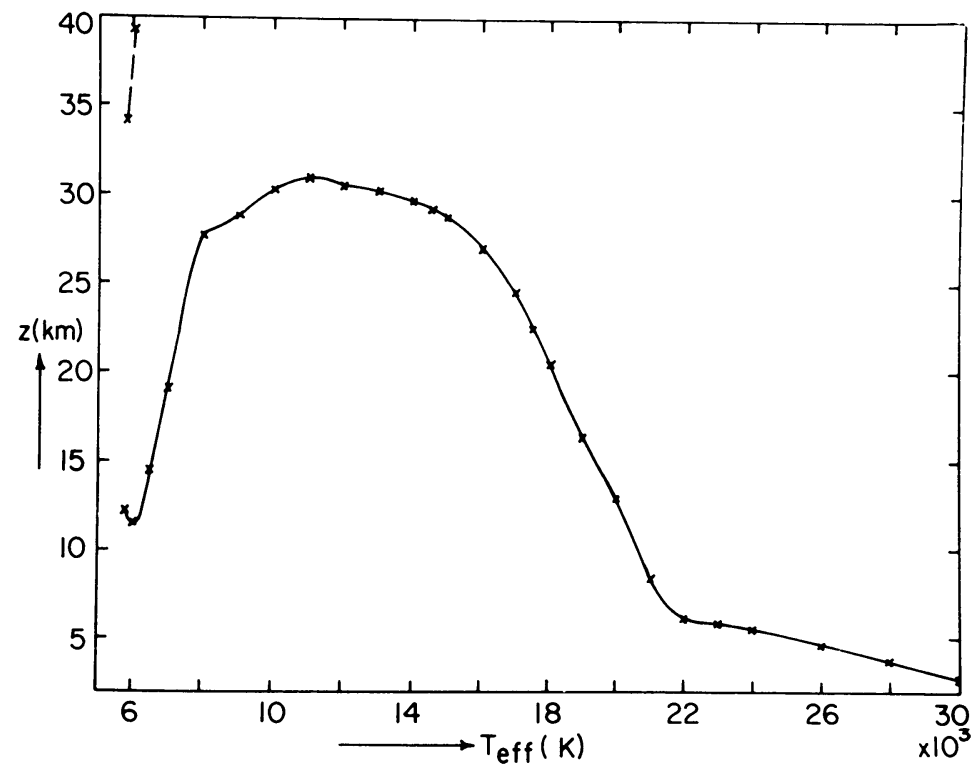

Fig. 2. The depth of the convection zone as a function of effective temperature. (The crosses correspond to the models which have been computed.) $g=10^{8} \mathrm{~cm} \mathrm{sec}-2$ has been used for all models. The short broken line corresponds to the separate lower convection zone which occurs in cool white dwarfs. 


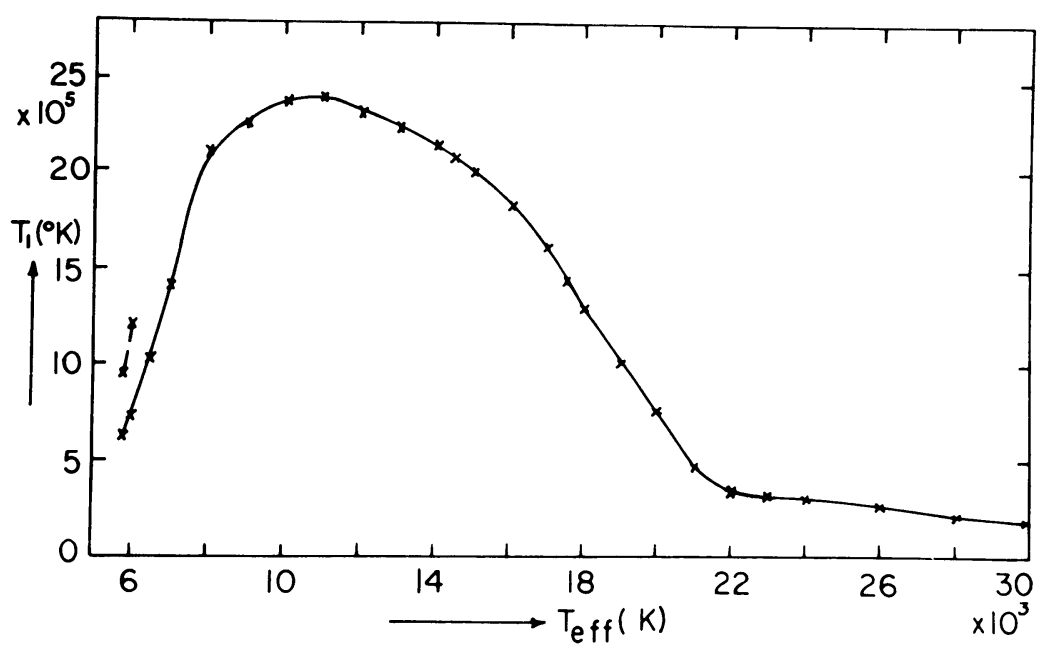

Fig. 3. The temperature at the lower boundary of the convection zone for white dwarfs of different effective temperatures. (The broken line has the same meaning as in Figure 2.)

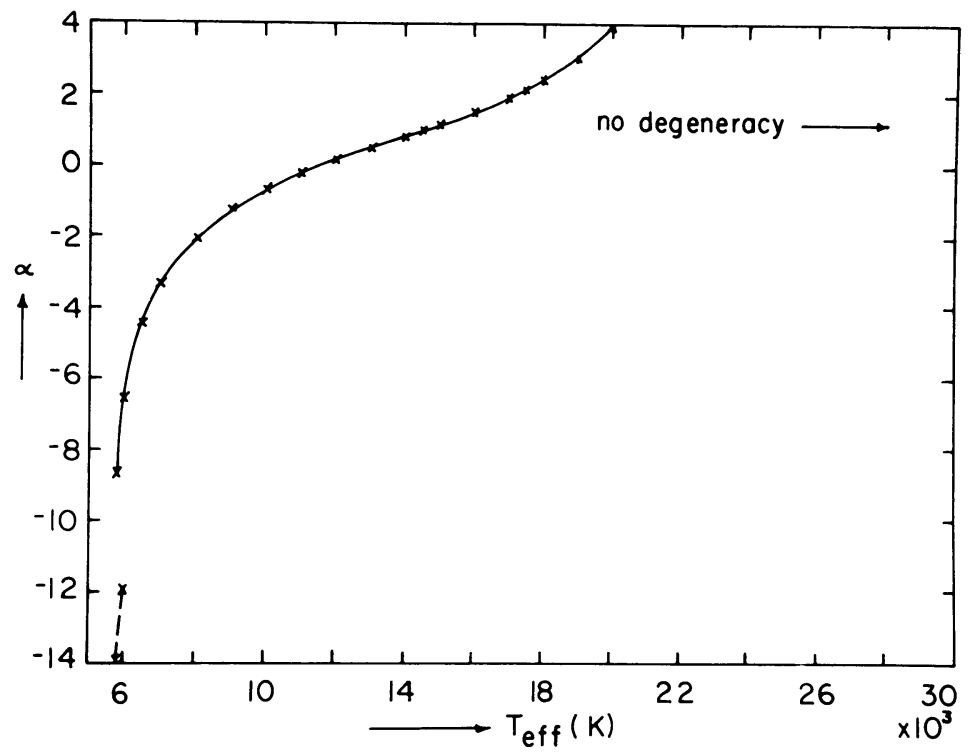

Fig. 4. The degeneracy parameter $\alpha=-\mu / k T$ at the lower boundary of the convection zone for white dwarfs of different effective temperatures. (The broken line has the same meaning as in Figure 2.)

(1) Beyond $T_{\text {eff }}=11000 \mathrm{~K}$ the thickness of the convection zone starts to decrease with increasing $T_{\text {eff }}$. For high $T_{\text {eff }}$ the outer convection zones become relatively thin.

(2) The degeneracy parameter $(-\alpha)$ at the bottom of the convection zone decreases rapidly with increasing $T_{\text {erf }}$. This means that for cool white dwarfs the convection zone reaches down to the 'transition point' (see e.g. Schwarzschild, 1958) to the almost 
isothermal core and consequently directly influences the temperature gradient in the whole nondegenerate envelope. In a hot white dwarf on the other hand the convection zone covers only a small fraction of the distance to the transition point.

One intriguing result of our computations is related to the convective velocities. As one might have expected the velocities are very low in cool white dwarfs. On the other hand, in hot white dwarfs with their much lower densities and their higher energy fluxes the convective velocities can become considerable. A value of $6.05 \mathrm{~km} / \mathrm{sec}$ is reached in a white dwarf with an effective temperature of $30000 \mathrm{~K}$. The velocity of sound at the same point in the same convection zone is $18.80 \mathrm{~km} / \mathrm{sec}$. This leads to a Mach number $M$ of 0.322 which is higher than the maximal Mach number occurring in the solar convection zone.

If we use the usual estimate of the acoustic energy flux

$$
H_{a c}=19 \varrho v_{\max }^{3} M^{5}
$$

(Lighthill, 1952; Bierman and Lüst, 1960) we find an acoustic energy flux of $1.5 \times 10^{11} \mathrm{erg} \mathrm{cm}^{-2} \mathrm{sec}^{-1}$ which has to be compared to $4.6 \times 10^{13} \mathrm{erg} \mathrm{cm}^{-2} \mathrm{sec}^{-1}$ for the total flux. These numbers show that hot white dwarfs have a considerable acoustic output and might easily develop chromospheres and coronae. In fact the acoustic flux in such a white dwarf is about twice as high as the total radiative flux in the sun. In view of the high gravitational field of the white dwarf we have to expect a corona which has a higher temperature than the solar corona. This follows from an estimate as suggested by Shklovsky (1965) who assumes that $\left(v_{\text {escape }} / v_{\text {thermal }}\right)^{2}$ is the same for all stellar coronae. From such an estimate we get temperatures of the order of $10^{8} \mathrm{~K}$ for white dwarf coronae. A qualitative extrapolation of Kuperus' (1965) results to stars with high $g$ indicates a temperature of only a few million degrees. It is interesting to note that such a hot white dwarf with a corona could have a relatively high ratio of X-ray emission to optical emission because of

(1) the relatively high ratio of acoustic to total flux in these objects

(2) the high bolometric correction which is required even if one neglects the radiation of the corona.

However, the X-ray radiation certainly cannot be strong enough to explain strong thermal X-ray sources like Sco X-1.

A further question which cannot yet be answered is whether some of the emission lines observed in some white dwarfs (see e.g. Greenstein, 1958, 1960) can be attributed to chromospheres heated by acoustic waves.

\section{References}

Bierman, L. and Lüst, R.: 1960, in Stellar Atmospheres (ed. by J. L. Greenstein), University of Chicago Press, Chicago.

Böhm, K. H.: 1968, Astrophys. Space Sci. 2, 375.

Böhm, K. H.: 1969, in Low Luminosity Stars (ed. by S. S. Kumar), Gordon and Breach, New York, p. 393.

Böhm, K. H.: 1970, Astrophys. J. 162, 919. 
Chandrasekhar, S.: 1939, An Introduction to the Study of Stellar Structure, University of Chicago Press, Chicago.

Greenstein, J. L.: 1958, in Handbuch der Physik 50 (ed. by S. Flügge), Springer-Verlag, Berlin, p. 161. Greenstein, J. L.: 1960, in Stellar Atmospheres (ed. by J. L. Greenstein), University of Chicago Press, Chicago, p. 676.

Greenstein, J. L.: 1969, Comments Astrophys. Space Phys. 1, 62.

Kuperus, M.: 1965, The Transfer of Mechanical Energy in the Sun and the Heating of the Corona, Reidel, Dordrecht.

Lighthill, M. J.: 1952, Proc. Roy. Soc. (London) 211, 564.

Rouse, C. A.: 1964, Astrophys. J. 139, 339.

Schwarzschild, M.: 1958, Structure and Evolution of the Stars, Princeton University Press, Princeton. Shklovskij, I. S.: 1965, Physics of the Solar Corona, Pergamon Press, Oxford.

Stewart, T. C. and Pyatt, K. D.: 1966, Astrophys. J. 144, 1203.

Van Horn, H. M.: 1970, Astrophys. J. (Letters) 160, 53.

Weidemann, V.: 1960, Astrophys. J. 131, 638. 